\title{
Comparison of two low-sensitivity urine pregnancy tests for confirming the success of early medical abortion
}

\author{
Sarah Louise Millar, ${ }^{1}$ Sharon Tracey Cameron ${ }^{1,2}$
}

${ }^{1}$ Chalmers Sexual Health Clinic, Edinburgh, UK

${ }^{2}$ Obstetrics and Gynaecology, University of Edinburgh, Edinburgh, UK

\section{Correspondence to} Professor Sharon Tracey Cameron, Chalmers Sexual Health Centre, Edinburgh EH3 9ES, UK; sharon.cameron@ ed.ac.uk

Received 16 July 2017 Revised 27 October 2017 Accepted 30 October 2017 Published Online First 25 November 2017

\begin{abstract}
Background We introduced a single-window low-sensitivity urine pregnancy test (LSPT) to replace a double-window LSPT (both 1000 IU hCG) for self-assessment of the outcome of early medical abortion (EMA) ( $\leq 63$ days gestation) 2 weeks later. We wished to compare assessment of outcomes of EMA with each LSPT.

Methods A retrospective review of the outcomes of EMA during 10 months' use of the doublewindow LSPT and the subsequent 10 months' use of the single-window LSPT to compare (i) detection of ongoing pregnancies and (ii) falsepositive and invalid results with each LSPT. Results 492 and 555 women self-assessed the outcome of their EMA with the double- and single-window LSPTS, respectively. Ongoing pregnancies were uncommon and occurred in $4 / 1047$ women $(0.4 \%)$. Two of these four women did not conduct a LSPT as they presented before the LSPT was due with scant bleeding or continuing pregnancy symptoms. False-positive LSPT results occurred in 6 (1.2\%) and 19 (3.4\%) double- and single-window LSPT tests, respectively $(P=0.0244)$. Invalid results were reported in 18 (3.6\%) and $6(1.1 \%)$ of doubleand single-window LSPT groups, respectively $(\mathrm{P}=0.01)$.

Conclusion The introduction of the singlewindow LSPT has not impacted on the detection of ongoing pregnancy or on contact with the service due to a positive or invalid LSPT. Services could consider use of either LSPT but should also place emphasis on informing women about the clinical signs and symptoms that suggest failed abortion
\end{abstract}

\section{INTRODUCTION}

Continuing pregnancy occurs in around $0.5-1 \%$ of cases of early medical abortion (EMA) ( $\leq 63$ days amenorrhea). ${ }^{1}$ Although the WHO advises that routine clinic follow-up is not necessary after first-trimester medical abortion, ${ }^{2}$ most

\section{Key messages}

- Abortion services should provide clear information to women undergoing early medical abortion (EMA) at home about the signs and symptoms of continuing pregnancy.

- Women with scant bleeding after EMA or persisting pregnancy symptoms should not delay in contacting their abortion provider.

- Many continuing pregnancies after EMA can be detected in the first week after abortion based on signs and symptoms alone.

- Use of either a single- or double-window low-sensitivity urine pregnancy test (detection limit 1000 IU hCG) can be used to exclude continuing pregnancy after EMA.

UK services have follow-up pathways in place that aim to exclude ongoing pregnancy. ${ }^{1}$ While ultrasound is accurate at detecting ongoing pregnancy there is a tendency to over-treat clinically asymptomatic retained products of conception seen on ultrasound soon after an abortion. ${ }^{1}$ This, together with non-attendance rates at follow-up as high as $84 \%$ and the financial implications of routine ultrasound follow-up, has meant that many services no longer routinely use it as a way of confirming successful abortion. ${ }^{3-5}$

Self-performed high- or low-sensitivity urine pregnancy tests (LSPT) conducted by women at home in conjunction with a telephone follow-up have been shown to be reliable at excluding ongoing pregnancy and are acceptable to women. ${ }^{6-10}$ Self-assessment (ie, no routine call from the abortion service) has also been shown to be effective for follow-up. ${ }^{11}$ In Scotland, the Edinburgh abortion service 
introduced self-assessment for follow-up after EMA in 2012. Women phone the abortion service if they have symptoms and signs of ongoing pregnancy, less than 4 days of bleeding and/or a positive or invalid self-performed LSPT (detection limit 1000 IU human chorionic gonadotropin (hCG)) at 2 weeks after misoprostol administration. ${ }^{11}$ Women sign a form stating that they agree to self-assessment and understand the test result and signs and symptoms that indicate when they should contact the service. A retrospective review of over 1700 women who had EMA at the Edinburgh abortion service found that $96 \%$ of women having EMA chose self-assessment. ${ }^{11}$

While many services use a LSPT as part of EMA follow-up, no studies have compared types of LSPT. The Edinburgh abortion service used Babycheck-1 duo (Quadratech Diagnostics Ltd, Epsom, UK) until October 2014. This was a double-window pregnancy test with 5 IU and 1000 IU hCG detection limits. For this test, women use a pipette to place a sample of urine into the corresponding well of the test cassette. There have been a small number of women with delayed presentation of a continuing pregnancy (presentation in mid-trimester) when using this test. ${ }^{6-8} 11$ In view of this, there was some concern that perhaps the doublewindow and the requirement to use a pipette were difficult for women to use and interpret. In October 2014, the Edinburgh abortion service switched to a LSPT with a single window (detection limit also 1000 IU hCG) called Check4 (Quadratech Diagnostics Ltd, Epsom, UK). This test is European conformity (CE) marked for home use and is conducted by holding the absorbent tip under a stream of urine or by placing it in a sample of urine. For the remainder of this article the Babycheck-1 duo test will be referred to as the double-window LSPT and the Check4 test as the single-window LSPT.

The main objective of this study was to determine if the switch from a double-window LSPT to the singlewindow LSPT impacted on the detection of ongoing pregnancy or on contact with the service due to a positive or invalid LSPT.

\section{METHODS}

A retrospective review of the Edinburgh abortion service database (Chalmers Centre, Edinburgh) was conducted for women having EMA between December 2013 and July 2015 (20 months). Some 1058 women chose to have an EMA during this time and 1047 (99\%) chose self-assessment as a way of confirming the success of EMA. A further 11 women (1\%) chose to have a planned post-abortion ultrasound scan. A total of 492 women had self-assessment in the 10 months before the change of LSPT (double-window LSPT: December 2013 to September 2014 inclusive) and 555 in the 10 months after the introduction of the new LSPT (single-window LSPT: October 2014 to July 2015 inclusive). The EMA regimen used by this service has previously been described; $200 \mathrm{mg}$ oral mifepristone is followed $24-48$ hours later with $800 \mu$ g misoprostol administered vaginally. ${ }^{11}$ The abortion service database routinely records demographics, gestation of pregnancy, reproductive history, outcome of abortion, contraception provision and contact made with the service. ${ }^{611}$ The outcome of EMA in the groups was determined based on a review of women's computerised national sexual health records and regional computerised hospital records, including maternity services (to capture failed EMA that may result in a delivery). Numbers of women contacting the service due to a positive or invalid LSPT were also recorded, together with details on detection of all ongoing pregnancies in both groups. Invalid results refer to the results where the woman reports that the test's control line had not appeared or they were unsure if it was a negative or positive result.

This project was approved by the local Sexual and Reproductive Health Quality Improvement Team and ethical approval was not required.

Statistical analysis was conducted using GraphPad software (GraphPad Software, La Jolla, California, USA) and the two groups were compared using Fisher's exact test. A P value of $<0.05$ was taken to be statistically significant.

\section{RESULTS}

The demographics of the 492 women in the doublewindow group and the 595 women in the singlewindow group detailed above are shown in table 1 . There were no statistically significant differences between the groups.

There were four ongoing pregnancies; one in the period of use of the double-window LSPT and three

Table 1 Demographics of women in both groups. Figures shown are number (\%) except for age

\begin{tabular}{lcc}
\hline Demographic & $\begin{array}{l}\text { Double- } \\
\text { window LSPT } \\
(\mathbf{n}=492)\end{array}$ & $\begin{array}{l}\text { Single- } \\
\text { window LSPT } \\
\mathbf{( n = 5 5 5 )}\end{array}$ \\
\hline Age range (years) & $16-47$ & $16-47$ \\
\hline Mean (SD) & $26.7(6.7)$ & $27.0(6.7)$ \\
\hline Gestation (days) & $345(70)$ & $383(69)$ \\
\hline$<49$ & $89(18)$ & $108(19)$ \\
\hline $50-56$ & $57(12)$ & $64(12)$ \\
\hline $57-63$ & $1(0.2)$ & $0(0)$ \\
\hline$>64$ & $210(43)$ & $207(37)$ \\
\hline Reproductive history & $158(32)$ & $190(34)$ \\
\hline Previous birth & $54(11)$ & $51(18)$ \\
\hline $\begin{array}{l}\text { Previous abortion } \\
\text { Previous } \\
\text { miscarriage }\end{array}$ & $0(0)$ & $4(0.8)$ \\
\hline Previous ectopic & \\
\hline LSPT, low-sensitivity pregnancy text. &
\end{tabular}


Table 2 Number and percentage of invalid, positive, total (invalid plus positive) LSPT results with double- and single-window LSPT

\begin{tabular}{lccc}
\hline LSPT result & $\begin{array}{l}\text { Double-window LSPT } \\
(\mathbf{n}(\%, 95 \% \mathrm{Cl}))(\mathbf{n}=492)\end{array}$ & $\begin{array}{l}\text { Single-window LSPT } \\
(\mathbf{n}(\%, 95 \% \mathbf{C l}))(\mathbf{n}=555)\end{array}$ & P value \\
\hline Invalid & $18(3.6,95 \% \mathrm{Cl} 2.2$ to 5.8$)$ & $6(1.1,95 \% \mathrm{Cl} 0.4$ to 2.4$)$ & 0.006 \\
\hline Positive & $6(1.2,95 \% \mathrm{Cl} 0.5$ to 2.7$)$ & $19(3.4,95 \% \mathrm{Cl} 2.2$ to 5.3$)$ & 0.024 \\
\hline Total & $24(4.9,95 \% \mathrm{Cl} 3.3$ to 7.2$)$ & $25(4.5,95 \% \mathrm{Cl} 3.0$ to 6.6$)$ & 0.772 \\
\hline LSPT, low-sensitivity pregnancy text. & &
\end{tabular}

in the period of use of the single-window LSPT. The rates of ongoing pregnancy in the double- and singlewindow phases were $0.2 \%$ and $0.6 \%$, respectively (difference not statistically significant). The woman with ongoing pregnancy in the double-window LSPT phase had an EMA at 6 weeks gestation and had a positive LSPT at 2 weeks but did not contact the abortion service with ongoing pregnancy symptoms until 5 weeks after her EMA. She had a surgical abortion at 12 weeks gestation. Of the three ongoing pregnancies in the period of use of the single-window LSPT, one woman presented at 4 weeks after EMA with pain. She had not done a LSPT and went on to have a surgical abortion at 11 weeks gestation. The other two women presented 4 and 5 days after EMA at 6 and 7 weeks gestation, respectively, due to minimal bleeding. They had therefore not reached the point at which they would have performed their LSPTs. One proceeded to repeat EMA and the other to surgical abortion.

A total of 24 women reported invalid results and returned to the abortion service for review (table 2). A statistically significant higher proportion of women in the double-window LSPT group reported invalid results compared with women in the single-window LSPT group $(\mathrm{P}=0.006)$. There were no continuing pregnancies in those with invalid test results. Twenty-two of these women (92\%) had ultrasound scans and the remaining two (8\%) had a repeat LSPT (negative) that was conducted by a healthcare professional in the abortion service. Three women (12.5\%) with invalid tests had ultrasonically visible tissue or clot (double-window LSPT group $\mathrm{n}=1$, single-window LSPT group $n=2$ ). One of these women had a surgical uterine evacuation.

A total of 25 women reported a positive LSPT result (table 2 ). Statistically significantly more women in the single-window LSPT group reported a positive LSPT result compared with women in the doublewindow LSPT group $(\mathrm{P}=0.024)$. All these women had an ultrasound scan. There was one ongoing pregnancy which was in the double-window LSPT group (see above). Of the women with positive tests, five (20\%) (double-window LSPT group $\mathrm{n}=1$, singlewindow LSPT group $n=4$ ) had intrauterine products of conception seen on ultrasound, which did not require intervention.

\section{DISCUSSION}

The switch from a double-window to a new singlewindow LSPT as part of self-assessment has not impacted on either the detection of ongoing pregnancies after EMA, or on contact rates with the service due to positive or invalid LSPT results. Ongoing pregnancies in both groups were detected because the women presented with symptoms of failed abortion. Two of the four ongoing pregnancies were detected within the first week after EMA (before the LSPT was due) due to reported minimal bleeding. The remaining women with ongoing pregnancies either did not do the test or failed to act for some time on a positive result. The switch to a single-window LSPT was associated with in an increase in reported false-positive results but a decrease in invalid results compared with the double-window LSPT. This meant that overall there were no differences between the two groups in terms of the number of women who returned to the abortion service for review after LSPT. From a cost point of view the single-window LSPT is more than twice the cost of the double-window LSPT (approximately $£ 5.30$ versus $£ 2.12$, but costs may vary according to quantity purchased and supplier used).

One might consider that a single-window LSPT would be easier to interpret than a double-window test. Our study was a retrospective database review and so it was not possible to determine whether women found the single-window LSPT easier to use and interpret. However, a study among women from a remote part of India reported that even those with low literacy skills found the double-window LSPT (used in the initial phase of our study) easy to use and interpret. ${ }^{12}$

The strengths of this study are that it included a large number of women and that the regional computerised records permitted us to identify all cases of ongoing pregnancy within the region. We cannot of course completely exclude the possibility that there may have been women with continuing pregnancies who moved out of the region and did not contact the service. However, we feel that this is unlikely, and the ongoing pregnancy rate that we observed in this study $(0.4 \%)$ is in keeping with that reported in the literature. ${ }^{1}$ Another limitation of our study is that it was a 'before and after' study design so we cannot exclude the possibility of residual confounding due to its non-randomised nature. In addition, it was a 
retrospective review and we did not actively follow-up the women, but assumed that those who did not contact our service had a negative LSPT. We are thus unable to calculate the sensitivity and specificity for each LSPT. Our study did not include women under the age of 16 years, vulnerable women or those with learning difficulties because they would generally not be suitable for self-assessment within our service. ${ }^{11}$

Self-performed LSPT, with or without telephone follow-up, has become routine practice in many services in the UK and Europe for the follow-up of women who have EMA. ${ }^{8} 11$

However, based on our findings and the high efficacy of EMA, one could argue that a routine LSPT at 2 weeks is not necessary for detecting ongoing pregnancy, as such pregnancies can be detected though the recognition of symptoms and signs before the LSPT is even due. Some women will not conduct a test even when provided with a LSPT at no cost; also others fail to act on a positive result. ${ }^{71}$ In addition, there are women who fail to attend a clinic visit for a confirmatory ultrasound scan for ongoing pregnancy, even when they are concerned enough to call the abortion service about a positive pregnancy test. ${ }^{11}$ In contrast, some studies have reported that ongoing pregnancies can be detected based on the LSPT test alone being positive. ${ }^{6711}$ In addition, it is known that women report that they value the reassurance of having a negative LSPT after EMA. ${ }^{13}$ Other workers have suggested that a better option may be the use of a multilevel pregnancy test (MLPT) that has a range of several thresholds for urine hCG and that can be used to determine the success of EMA in some women as early as 3 days after mifepristone administration. ${ }^{14}$ Studies from a number of countries have shown that a MLPT with five windows is reported to be easy to use and interpret by women undergoing EMA. ${ }^{14}$ Whether or not a MLPT at this early stage has any advantage over advising women to contact the abortion service if they have scant bleeding or pregnancy symptoms in the first weeks after EMA remains to be determined.

\section{CONCLUSION}

Abortion services that use LSPT as part of self-assessment should also provide women with clear verbal and written information on the signs and symptoms that would suggest ongoing pregnancy, and encourage them to make contact without delay in such circumstances and not wait until the LSPT is due. In addition, women who do have a positive or invalid LSPT should likewise be encouraged to act on those results.

Acknowledgements The authors are grateful to Anne Johnstone (research nurse) for assistance with data collection.

Contributors SLM conducted the review and wrote the paper. STC supervised SLM and made changes to the paper and approved the final version.
Competing interests None declared.

Provenance and peer review Not commissioned; externally peer reviewed.

(C) Article author(s) (or their employer(s) unless otherwise stated in the text of the article) 2018. All rights reserved. No commercial use is permitted unless otherwise expressly granted.

\section{REFERENCES}

1 Royal College of Obstetricians and Gynaecologists. The care of women requestinginduced abortion. Guideline number 7 . 2011. www.rcog.org.uk (accessed 16 Jul 2017).

2 World Health Organization. Safe abortion: technical and policy guidance for health systems. 2nd edn. Geneva, Switzerland: World Health Organization, 2012.

3 Astle H, Cameron ST, Johnstone A. Comparison of unscheduled re-attendance and contraception at discharge, among women having the final stage of early medical abortion at home and those remaining in hospital. J Fam Plann Reprod Health Care 2012;38:35-40.

4 Grossman D, Ellertson C, Grimes DA, et al. Routine follow-up visits after first-trimester induced abortion. Obstet Gynecol 2004;103:738-45.

5 Bracken H, Lohr PA, Taylor J, et al. RU OK? The acceptability and feasibility of remote technologies for follow-up after early medical abortion. Contraception 2014;90:29-35.

6 Cameron ST, Glasier A, Dewart H, et al. Telephone followup and self-performed urine pregnancy testing after early medical abortion: a service evaluation. Contraception 2012;86:67-73.

7 Michie L, Cameron ST. Simplified follow-up after early medical abortion: 12-month experience of a telephone call and self-performed low-sensitivity urine pregnancy test. Contraception 2014;89:440-5.

8 Oppegaard KS, Qvigstad E, Fiala C, et al. Clinical followup compared with self-assessment of outcome after medical abortion: a multicentre, non-inferiority, randomised, controlled trial. Lancet 2015;385:698-704.

9 Ngoc NT, Bracken H, Blum J, et al. Acceptability and feasibility of phone follow-up after early medical abortion in Vietnam: a randomized controlled trial. Obstet Gynecol 2014;123:88-95.

10 Perriera LK, Reeves MF, Chen BA, et al. Feasibility of telephone follow-up after medical abortion. Contraception 2010;81:143-9.

11 Cameron ST, Glasier A, Johnstone A, et al. Can women determine the success of early medical termination of pregnancy themselves? Contraception 2015;91:6-11.

12 Iyengar K, Paul M, Iyengar SD, et al. Self-assessment of the outcome of early medical abortion versus clinic follow-up in India: a randomised, controlled, non-inferiority trial. Lancet Glob Health 2015;3:e537-45.

13 Constant D, Harries J, Daskilewicz K, et al. Is self-assessment of medical abortion using a low-sensitivity pregnancy test combined with a checklist and phone text messages feasible in South African primary healthcare settings? A randomized trial. PLoS One 2017;12:e0179600.

14 Raymond EG, Shochet T, Blum J, et al. Serial multilevel urine pregnancy testing to assess medical abortion outcome: a metaanalysis. Contraception 2017;95:442-8. 